\title{
Literaturbericht
}

Tom Mannewitz

\section{Regionale politische Kultur zwischen Symbolanalysen und Surveydaten - methodische, konzeptionelle und geografische Desiderate der deutschen Forschung nach 1990}

\section{Einleitung}

Das Interesse an politischer Kultur ist ungebremst. Nachdem die Gründungsväter Sidney Verba und Gabriel Almond mit „The Civic Culture“ (1963) vor über einem halben Jahrhundert dieses neue Forschungsgebiet theoretisch wie empirisch erschlossen hatten, breitete sich die wissenschaftliche Neugier an der „subjektiven Dimension von Politik" (Greiffenhagen/Greiffenhagen 1993: 23) zwar langsam, aber stetig aus. Das Verdienst der beiden US-Amerikaner war es, die politikwissenschaftliche Aufmerksamkeit auf einen - in seiner Elaboriertheit neuen Topos gerichtet zu haben, der Schwächen institutionenbasierter Ansätze zur Erklärung von Regimewandel auszufüllen versprach. Es sollte nicht überraschen, dass sich ähnliche, von solcherlei intellektuellen Vorarbeiten befruchtete Konzepte aus dem angloamerikanischen Raum rasch Bahn brachen. Von ihrer Prägekraft bis heute nichts verloren haben etwa das Unterstützungskonzept David Eastons (1965 a, 1965 b, 1975), der nach Legitimität und Effektivität unterscheidende Ansatz Seymour Martin Lipsets (1963: 45 ff.) und die maßgeblich von Ronald Inglehart $(1977,1989)$ vorangetriebene Wertewandelforschung.

Der neue politikwissenschaftliche Trend aus den USA blieb nicht ohne Wirkung für die Sozialwissenschaft diesseits des Atlantik: Hiesige Autoren rezipierten die jüngeren theoretisch-konzeptionellen Ansätze zuhauf - teils, um sie einer empirischen Überprüfung in Deutschland zu unterziehen (Fuchs 1989; Gabriel 1987), teils, um ihre konzeptionelle Stichhaltigkeit anzufechten (Rohe 1987, 1990). Eine gewisse babylonische Sprachverwirrung ließ darum nicht lange auf sich warten: „One clear difficulty is that political culture has almost as many defi- 
nitions as authors who employ it" (Reisinger 1995: 334). Gleichwohl: Die Aufregung über die uneinheitliche Verwendung des Terminus hat sich mittlerweile ein wenig gelegt: Quantitativ-umfragebasierte Studien (Keil/van Deth 2012), die politische Kultur als Meinungs-, Einstellungs- und Wertemuster begreifen, haben ebenso ihren Platz gefunden wie qualitativ-hermeneutische - teils kulturwissenschaftlich inspirierte - Betrachtungen (Salzborn/Diehl 2013), die politische Symbole, routinisierte Handlungsmuster und historische Entwicklungspfade in den Mittelpunkt ihrer Aufmerksamkeit rücken, ohne dass jemand ernsthaft daran Anstoß nimmt: „Festzuhalten bleibt, dass dem Problem der ,zu breiten Begriffsverwendung' durch eine letzte, endgültige Entscheidung kaum beigekommen werden kann. [...] Forschungspraktisch wird es wohl eher so sein, dass jeder Forscher den von ihm verwendeten Begriff von politischer Kultur für seine Untersuchung explizit offen legen sollte" (Pickel/Pickel 2006: 111).

Ganz gleich, ob eher qualitativ oder eher quantitativ: Hier wie da hat der methodologische Nationalismus (Chernilo 2006; Jeffery/Wincott 2010; Martins 1974) erstaunliche Beharrungskräfte entfaltet - erkennbar daran, dass politischkulturelle Analysen noch immer zu einem Großteil auf gesamtgesellschaftliche Betrachtungen hinauslaufen. Die Binnendifferenzierung wird geradezu stiefmütterlich behandelt: „Within the political culture literature comparatively little attention is dedicated to studying diversity within states" (Henderson $2010 \mathrm{a}$ : 470). Dieses Urteil einer intimen Kennerin verschiedenster subnationaler politischer Kulturen (u. a. Kanada, Großbritannien) trifft auf den deutschen Zweig der Politische-Kultur-Forschung allerdings nur teilweise zu, denn mit der Wiedervereinigung am 3. Oktober 1990 rückte die subnationale Heterogenität der hiesigen politischen Kultur ins Scheinwerferlicht der Sozialwissenschaft, die seither regelmäßig den Gemeinsamkeiten und Unterschieden zwischen Ost- und Westdeutschland auf den Grund geht. Durch dieses scheinbar vom Gegenstand aufgedrängte Forschungsdesign gerieten jedoch politisch-kulturelle Friktionen in Vergessenheit, die jenseits des Ost-West-Unterschieds liegen. Die mit dem bis heute dominierenden Ost-West-Vergleich einhergehenden - und von der Politikwissenschaft weitgehend ignorierten - Probleme zu erläutern ist Ziel des Forschungsüberblicks. Im Brennpunkt befindet sich jedoch die Frage, wie es um jenen Zweig bestellt ist, der sich mit dem Paarvergleich nicht zufrieden gibt.

Der terminologischen Grenzziehung schließt sich ein Überblick über die im Lichte der staatlichen Wiedervereinigung stehenden Forschung an. Durch den 3. Oktober 1990 erhielt der Ost-West-Vergleich, der bis heute nahezu als Synonym für Politische-Kultur-Forschung in Deutschland gilt, seine Daseinsberechtigung. Allerdings sind ihm gravierende Probleme zu eigen, die für eine Ablösung 
durch Vergleichsstudien auf anderen subnationalen Analyseebenen sprechen. Eine solche, scheinbar geringfügige Änderung im Forschungsdesign vermeidet zahlreiche Mängel des Ost-West-Vergleichs, findet aber - zumindest in Deutschland - immer noch allenfalls geteilte Aufmerksamkeit: Während Länderkunden, die sich der regionaltypischen Symbole, Riten und Traditionen annehmen, in den vergangenen 20 Jahren einen gewissen - wenngleich sanften - Bedeutungsschub erfahren haben (nicht zuletzt, weil der Bedarf aufgrund fünf - mit Berlin sechs „,neuer" Länder gestiegen ist), fristen subnationale komparative Analysen bis heute ein Schattendasein.

\section{Bedeutungen „regionaler politischer Kultur“}

Regionale politische Kultur ist nicht gleich regionale politische Kultur. Der Oberbegriff hierzu lautet politisch-kulturelle Heterogenität (Almond/Verba 1965: 26 f.; Henderson 2007: $4 \mathrm{f}$.). Der Begriff bezeichnet hier das Gegen- und Miteinander unterschiedlicher politischer Lager in einem Land (etwa Demokraten und Republikaner in den USA - horizontale Heterogenität), dort die Unterschiedlichkeit der politischen Eliten- und Soziokultur (vertikale Heterogenität - Rohrschneider 1999). Die dritte Bedeutung - relevant für die Erforschung regionaler politischer Kultur - kreist um regionale Vielfalt: regionale politische Kulturen und - aus anderer Perspektive - politisch-kulturelle Regionen. Damit sind - je nach Forschungstradition - geografische Konzentrationen politischer Meinungen, Einstellungen und Werte, Vorstellungen, Traditionen, Symbole, Riten u. Ä. gemeint.

Da „Region“ sich auf zwei Dinge beziehen kann, beschreibt „regionale politische Kultur" gleichfalls zwei verschiedene Sachverhalte: „When we speak of regional political cultures we should [...] distinguish between sub-state regional cultures and state regional cultures that exist within supra-state groupings" (Henderson 2007: 8). Wer sich, indem er auf Makro- bzw. Weltregionen blickt, im Bereich der Area-Studies bewegt, der hat transnationale politische Kulturen, mithin länderübergreifende Gemeinsamkeiten im Sinn (Blatter 2004; Caramani/Wagemann 2005; Inglehart/Carballo 1997). Unterhalb der nationalstaatlichen Ebene treten hingegen subnationale politische Kulturen und politisch-kulturelle Mikroregionen zutage. Der zugehörige Forschungszweig widmet sich der Ausdifferenzierung nationaler politischer Kulturen in regionaltypische Muster, die sich qualitativ wie quantitativ voneinander unterscheiden. Angesichts eines relativ eng umgrenzten Themengebietes frappiert hier das erstaunliche Maß an methodischer, theoretischer und konzeptioneller Vielfalt. 


\section{Der Ost-West-Vergleich nach 1990}

Deutscher politischer Kultur auf den Grund zu gehen, heißt seit etwa einem Vierteljahrhundert, „die“ politische Kultur „des“ Westens zu der „des“ Ostens ins Verhältnis zu setzen. Hatten auf subnationaler Ebene vormals einzelne Länder oder Landstriche zumindest vereinzelt im Brennpunkt gestanden (Landeszentrale für politische Bildung Baden-Württemberg 1985; Berg-Schlosser/Schissler 1987: 259 ff.), lieferte die Wiedervereinigung gleichsam ,natürliche“ Vergleichsfälle. Die einzigartige Situation ermöglichte quasi-experimentelle Studien, in denen sich die Nachwirkungen einer planwirtschaftlich sozialistischen Diktatur und einer tiefgreifenden Transformation auf eine Gesellschaft abschätzen ließen. So schien es ganz natürlich, dass „Ost“ und „West“ fortan zentrale Analysetopoi bei der Erforschung regionaler politischer Kultur waren.

Die Frage, die unzähligen Beiträgen in Sammelband-,$^{1}$ Aufsatz- ${ }^{2}$ und Monografieform (Greiffenhagen/Greiffenhagen 1993; Pickel 2002) den Anstoß gab, der „,inneren Einheit“ regelmäßig den Puls zu fühlen, lautete: „Gibt es in Deutschland eine oder zwei politische Kultur(en)?" Obwohl mittlerweile ein wenig abgeebbt, reißt der Fluss an aktualisierenden Publikationen bis heute nicht ab. ${ }^{3}$ Nicht unwesentlich daran beteiligt sein dürfte die Konfliktträchtigkeit der Leitfrage, hat sie doch bis heute im Großen und Ganzen zwei wissenschaftliche Positionen hervorgebracht: Der Mehrheit, die noch größere Hürden bei der „,inneren Einheit“ Deutschlands diagnostiziert, ${ }^{4}$ steht eine Minderheit gegenüber, die diesen Zustand seit geraumer Zeit erreicht ansieht (Ahbe/Gibas 2000; Veen 2012). Es fehlt nicht an vermittelnden Wissenschaftlern, die Gemeinsamkeiten wie Unterschiede anerkennen. ${ }^{5}$

Gemein ist den meisten Ansätzen nicht nur ihr quantitativer Einschlag, der sich am Rückgriff auf Umfragedaten bemerkbar macht, sondern auch ihre Neigung zu partiellen Analysen: Statt politisch-kulturelle Gesamtbetrachtungen haben in jüngerer Vergangenheit vor allem Studien zu einzelnen Aspekten das Licht der Welt erblickt. Fragt dieser Beitrag nach dem Vertrauen in Bundestag, Regierung und Verfassungsgericht, schenkt jene Analyse den Haltungen zur Demokratie-Idee

1 Beyme/Niedermayer 1994; Falter/Gabriel/Rattinger 2000; Fuchs/Roller/Weßels 2002; Gabriel/ Falter/Rattinger 2005; Meulemann 1998; Pickel/Pickel/Walz 1998.

2 Fuchs/Gabriel/Völk1 2002; Fuchs/Roller/Weßels 1997; Pollack 1999; Yoder 2000.

3 Beetz u. a. 2014; Bundesministerium für Wirtschaft und Energie 2015; Hollenstein 2012; Schneider 2013; Veen 2013.

4 Bahrmann/Links 2005; Greiffenhagen/Greiffenhagen 2000; Feist/Liepelt 1994; Reissig 2000; Schroeder 2000.

5 Gabriel 2005; Jesse 2008; Klages/Gensicke 1992; Patzelt 2008. 
und zur Demokratierealität in Deutschland Aufmerksamkeit; wird hier die Unterstützung von Spitzenpolitikern in Ost und West auf Gemeinsamkeiten wie Unterschiede abgeklopft, ist es dort das Meinungsbild bei den Aufgaben des Sozialstaates. Ein solcher Flickenteppich aus unverknüpften Teilergebnissen kommt an eine systematische Betrachtung nicht heran - und seien die Analysen noch so kenntnis- und aufschlussreich. Eine ganzheitliche Gegenüberstellung der politischen Kulturen von Ost und West auf Basis aktueller Daten bzw. im Längsschnitt steht aus.

Die Debatte verläuft zudem unbefriedigend: Ihr Ende ist selbst nach mehr als 20 Jahren noch immer nicht in Sicht. Für diese fachliche Zerstrittenheit sind allerdings weder die unterschiedlichen Facetten politischer Kultur verantwortlich, auf die sich Forscher beziehen, noch variierende Frageformulierungen in den Surveys oder gar die Kurzlebigkeit politischer Meinungen und Einstellungen. Vielmehr sind drei Defizite dafür in Verantwortung zu nehmen:

\section{a) Defizit 1: Externe Maßstäbe werden nicht herangezogen}

Durch den Rückgriff auf externe Maßstäbe - etwa den Vergleich mit anderen politisch-kulturell gespaltenen Ländern (Belgien, Großbritannien, Kanada, Italien, Spanien etc.) - ließen sich Friktionen zwischen Ost und West in ihrer Größenordnung relativieren. Ein - leider seltenes - Beispiel guter Praxis bietet Christoph Schneiders (2013) Dissertation, die zum Schluss kommt, Disparitäten zwischen „alten“ und „neuen Ländern“ höben sich nur unwesentlich von jenen innerhalb Großbritanniens, Spaniens, Italiens und Belgiens ab. Es scheint, die Nabelschau verstelle zu häufig den Blick über den Tellerrand.

\section{b) Defizit 2: Statistische Verfahren spielen kaum eine Rolle}

Strukturerkennende und -prüfende Analyseinstrumente wie Cluster- oder Varianzanalysen, ${ }^{6}$ die in der Politische-Kultur-Forschung kaum verwendet werden, vermögen Scheidelinien innerhalb des Ostens/Westens bzw. quer zur Ost-WestDichotomie verlaufende freizulegen, die den bekannten Gegensatz in den Schatten stellen. So ist es keineswegs ausgemacht, dass die Gemeinsamkeiten im Osten (bzw. im Westen) größer ausfallen als zwischen beiden Landesteilen. Es ist eben-

6 Clusteranalysen könnten bei Umfragen zustande gekommene Mittel- und Anteilswerte der Bundesländer bei einzelnen Items ins Verhältnis zueinander setzen, Mittelwertvergleiche (t-Test) könnten auf Individualdaten- oder Bundesländerebene prüfen, ob zwischen Ost und West ein signifikanter Unterschied besteht, Varianzanalysen könnten auf Individualdatenebene Differenzen zwischen den Ländern ans Tageslicht bringen. 
falls ungeklärt, ob die beiden Landesteile überhaupt zentrale Untersuchungskategorien darstellen.

Beide Defizite - die fehlende Relativierung wie der ausbleibende Gebrauch statistischer Instrumentarien - sorgen bei der Einordnung von Ost-West-Unterschieden für ein gewisses Interpretationsvakuum, das nicht wenige Wissenschaftler mit „politische[m] Zweckoptimismus bzw. Zweckpessimismus“ (Greiffenhagen/ Greiffenhagen 2000: 180) füllen. Darum ist die Debatte um den politisch-kulturellen Graben zwischen Ost und West „bis heute nicht zuletzt geprägt von den politischen Positionen der Beteiligten bzw. einer gewissen Ratlosigkeit beim Rückgriff auf das Theorieangebot der politischen Kultur-Forschung" (Sturm 2004: $320)$.

\section{c) Defizit 3: Die Wissenschaft geht von zwei weitgehend homogenen Landes- teilen aus}

Dass die Politische-Kultur-Forschung cluster- und varianzanalytische Verfahren stiefmütterlich behandelt, dürfte nicht unwesentlich an ihrer impliziten Annahme, Ost und West seien zwei in sich geschlossene Landesteile, liegen. Um politischkulturelle Regionalisierung nachzuweisen, führt sie darum ausschließlich Paarvergleiche durch. Um aber zu zeigen, dass die Differenzen zwischen Ost und West tatsächlich maßgeblich sind und von einem einheitlichen Osten und einem einheitlichen Westen gesprochen werden kann, braucht es stets zwei Vergleiche: einen interregionalen (zwischen Ost und West) und einen intraregionalen (innerhalb des Ostens und des Westens). Spannungen innerhalb beider Landesteile, die größer ausfallen als Spannungen zwischen ihnen, widersprächen der These von zwei politischen Kulturen. „Der Vorteil dieser weitergehenden Differenzierung liegt v. a. darin, dass Unterschiede in den Einstellungen und Bewertungen der jeweiligen Bevölkerungsanteile - anders als bei den Aggregatdaten - als mögliche Artefakte des Ost-West-Splitts enttarnt werden können. Wertedifferenzen stellen sich dann mitunter nicht mehr als Ost-West-Unterschiede, sondern (auch) als Nord-Süd-Unterschiede oder entlang anderer sozialstruktureller und regionaler Linien verlaufender Differenzen dar." (Pickel 2011: 388)

\section{Regionale politische Kultur zwischen Survey-Studien ...}

Eine Möglichkeit, der Regionalisierung politischer Kultur auf den Grund zu gehen, ohne zugleich den Defiziten der Ost-West-Forschung aufzusitzen, bietet ein Politische-Kultur-Vergleich unterhalb der Nationalstaats- und der Ost-West-Ebene. Innerhalb dieses - äußerst überschaubaren - Forschungssegments dienen - 
wie auch bei Studien zu anderen Staaten - mehrheitlich Gebietskörperschaften, sprich: die Bundesländer, als Analyseeinheiten. Dafür spricht deren prägender Einfluss auf die politischen Orientierungen gegenüber dem zentralen politischen Entscheidungssystem auf nationaler Ebene. Ein solcher Einfluss des Föderalismus auf die Orientierungen gegenüber dem nationalen politischen System vermittelt sich vor allem über die Institutionen: ${ }^{7},[$ I] nstitutions bring with them norms of governance that become the boundaries of new political cultures. New sub-state institutions are thus able to create new political cultures, and thus can lead to greater variation of political cultures within a single state." (Henderson 2007: 6). Robert D. Putnams (1993) Studie über Sozialkapital in Italien unterstreicht diese Wirkmächtigkeit.

Dabei fällt der Effekt umso stärker aus, je weiter die föderalen Strukturen institutionell vervollständigt sind (Breton 1970): „A region specific media, publishing in a regional language, reporting on the workings of a regional government, creates a regional reference to political life and raises the likelihood of generating regional-specific approaches to politics and policy, citizenship and community membership.“ (Henderson 2010 b: 441).

Außerdem beeinflusst der Föderalismus politische Orientierungen auf mittelbare Weise (Cohen 1996): ,[I]ndividuals understand their own reality through the lens of their own particular circumstances." (Henderson 2010 b: 441) ${ }^{8}$ Föderale Strukturen variieren die individuellen Umwelten der Gesellschaft demnach ganz verschiedentlich - zumal in Deutschland: über die Demografie-, die Bildungs-, die Wissenschafts-, die Sicherheits-, die Wirtschafts- und Standortförderungspolitik, die Pflege regionaler Bräuche, Feste und Feiertage.

Wie ist es nun um diesen Forschungszweig bestellt? Kurzum: nicht allzu gut. Eine der wenigen subnational vergleichenden Betrachtungen aus dem Bereich der Meinungs-, Einstellungs- und Werteforschung - Surveystudien dominieren im komparativen Sektor der Regionale-Politische-Kultur-Forschung - haben Markus Freitag und Richard Traunmüller (2008) mit ihrer Sozialkapitalstudie vorgelegt. Ihnen zufolge weisen Nord-, Mittel- und Süddeutschland eine hohe soziale Netzwerkdichte auf - anders als der unter den sozialistischen Hinterlassenschaften leidende Osten. Eine Sekundäranalyse der Bertelsmann-Stiftung (2014) sowie eine empirische Studie von Markus Freitag und Till Heinsohn (2011) bestätigen dieses Muster, wenn sie dem Osten in der DDR-Vergangenheit wurzelnde Mängel beim zwischenmenschlichen Vertrauen attestieren. Mit Blick auf die sogenannten Rezi-

7 Elkins/Simeon 1974; Henderson 2010 a; Lieske 2010; Lepsius 2009: 71; McGrane/Berdahl 2013.

8 Alm u. a. 2001. 
prozitätsnormen - also die Haltung, dass man für andere etwas tut, ohne dafür eine Gegenleistung zu erwarten - schneiden die östlichen Länder hingegen besser $\mathrm{ab}$, die südlichen schlechter. Hier scheint das sozialistische Erbe ebenfalls durch mit für die Demokratie erfreulicheren Konsequenzen.

Nicht weniger heterogen fällt der Anblick der Gesellschaft aus, wenn es um die postmaterialistische Wende geht (Mannewitz 2012). Zu den „Vorreitern“ der „stillen Revolution“ gehören demnach Rheinland-Pfalz und Hessen sowie die „Nordlichter“ Bremen, Hamburg und Schleswig-Holstein, zu den „Schlusslichtern" Baden-Württemberg, Berlin-West, Mecklenburg-Vorpommern, Sachsen und Sachsen-Anhalt. In allen übrigen Bundesländern - Bayern, Berlin Ost, Brandenburg, Niedersachsen, Nordrhein-Westfalen, Saarland, Thüringen - gibt es ähnlich viele Materialisten wie Postmaterialisten.

Was die Effektivität und Legitimität der Demokratie angeht (Seymour M. Lipset), ist das Bundesgebiet ebenfalls durch Vielfalt geprägt (Mannewitz 2013). Die Akzeptanz der Demokratie-Idee fällt - so die für den Bestand des politischen Systems überaus erfreuliche Nachricht - überall ausnehmend hoch aus. Allenfalls die Bundeshauptstadt weicht ein wenig vom Rest der Republik ab - allerdings nur wenig. Die Zufriedenheit mit den Leistungen der Demokratie hingegen trennt die Spreu vom Weizen: In strukturschwachen Gegenden zeigen sich die Menschen deutlich unglücklicher mit der Performanz des demokratischen Systems als in prosperierenden Regionen. Aus diesem Grund fällt die Demokratieeffektivität in den „,neuen Bundesländern“ und in Bremen niedriger aus als im Rest der Republik - Wasser auf die Mühlen jener, die eine Reform des Länderfinanzausgleichs zugunsten schwächelnder Regionen fordern.

Wer solche Einzelbetrachtungen beiseitelässt und versucht, die regionalen politischen Kulturen in Gänze zu beschreiben, dem offenbaren sich sanfte, gleichwohl nicht wegzudiskutierende Differenzen: So scheiden die demokratisch-konstitutionellen Wertorientierungen die Länder - zum Teil stark - voneinander (Mannewitz 2015). Das meint einerseits die variierende Übereinstimmung mit dem demokratischen Verfassungsstaat: Wahlen und Gewaltenteilung, Rechtsstaat, Partizipationsund Grundrechte, Verfassungsgerichtsbarkeit, Staatlichkeit. Andererseits schließt dies die Vorstellungen von einer ,guten“ konstitutionellen Demokratie ein. Diese gestattet schließlich ganz unterschiedliche institutionelle Ausprägungen - etwa mit Blick auf den Föderalismus, Umfang und Art plebiszitärer Elemente und den Umgang mit politischem Extremismus. Je nach dem, worum es sich gerade handelt, vertreten die Deutschen aus Ost und West, Nord und Süd ganz unterschiedliche politische Werte. Wer versucht, sie zu einem Gesamteindruck zu verdichten, dem offenbaren sich - grosso modo - drei politisch-kulturelle Regionen: eine 
eher libertär-konstitutionelle im Süden, eine eher demokratisch-konstitutionelle im Nordwesten, eine eher sozialistisch-revolutionäre im Osten. Bemerkenswert: Der Osten bildet keine Einheit, denn einerseits heben sich Mecklenburg-Vorpommern und Sachsen-Anhalt (trotz einiger Parallelen) bei vielen Wertefragen signifikant von den anderen „,neuen Ländern“ ab, sodass sie je einen Typus sui generis repräsentieren, andererseits ähnelt Thüringen den Ländern Niedersachsen, Nordrhein-Westfalen und Schleswig-Holstein stärker als seinen östlichen Nachbarn. Für dieses politisch-kulturelle Amalgam zeichnen das Ineinandergreifen, die Überspülung und die Verstärkung unterschiedlichster Einflüsse verantwortlich, deren zwei Hauptgrößen die Konfessionalität eines Landes (protestantisch, katholisch, keine) und eine längere demokratische Erfahrung (DDR versus Bundesrepublik) sind.

Nun liegt der Blick auf die Bundesländer (oder - in anderen Staaten - auf Kantone, Wojewodschaften, Federal States usw.) bei einem subnationalen Vergleich auf der Hand. Das heißt jedoch nicht, andere Regionen könnten nicht ebenfalls Gegenstand wissenschaftlicher Auswertung sein. Dies trifft insbesondere für kleinteiligere Gebiete - Kommunen - und die entsprechenden Orientierungen ihnen gegenüber zu. Allerdings hat eine vergleichende Forschung auf diesem Gebiet, die ihren Namen verdient, in Deutschland nicht so recht Fuß fassen können. Abgesehen von einem Versuch Oscar W. Gabriels aus den frühen 1990er Jahren, der nicht zuletzt aufgrund fehlender Daten bescheiden ausfällt, ist dem Verfasser keine vergleichend angelegte Betrachtung bekannt. Gabriel diagnostiziert eine leidlich gute Zufriedenheit der Deutschen mit ihren Kommunalstrukturen und kann keine partizipativen Defizite, geschweige denn größere Unterschiede zu den Orientierungen gegenüber dem Nationalstaat erkennen. Kurzum: „Staat und Gemeinde bilden für [die Deutschen] eine Einheit.“" (Gabriel 1994: 244) Der wenig spektakuläre Befund ist allerdings schon über 20 Jahre alt und wurzelt auf keiner allzu breiten Datenbasis.

Zwei Dinge führen die - dünn gesäten - subnationalen Politische-Kultur-Vergleiche vor Augen. Erstens: Die These zweier politischer Kulturen erscheint nach über 20 Jahren unterkomplex, die Annahme zweier homogener Landesteile, auf denen sie beruht, kaum haltbar. Angesichts der politisch-kulturellen Binnendifferenzierung wirkt der Ost-West-Vergleich anachronistisch. Er ist nicht nur außerstande, zu klären, ob es so etwas wie ,innere Einheit" zwischen Ost und West gibt (weil dafür kein intersubjektiv nachvollziehbarer Maßstab herangezogen wird), sondern das Forschungsdesign überdeckt auch aufkeimende Friktionen zwischen den Ländern. Zweitens: Der subnationale Vergleich ist weithin ein Desiderat der hiesigen Politische-Kultur-Forschung. Während er etwa zum Standardinventar der 
vergleichenden Regierungslehre zählt, erfährt er in der politischen Soziologie nur geringe Aufmerksamkeit (Freitag/Vatter 2010). Diese hängt nach wie vor am OstWest-Vergleich - woran die geringe Verfügbarkeit „repräsentativer“ Fallzahlen auf Länderebene nicht ganz unschuldig sein dürfte (Freitag/Fritz/Vatter 2010: 13; Westle 2009: 536).

\section{5. ... und Symbolanalysen}

Neben den auf Umfragestudien basierenden Komparativstudien entfaltete sich in den letzten Jahrzehnten eine zweite Tradition, die der Erforschung regionaler politischer Kultur in der Bundesrepublik ihre Gestalt verliehen hat: hermeneutische Analysen mit stärker historisch-geisteswissenschaftlichem Bezug. Die Trennung zwischen beiden Strängen verläuft - vermutlich nicht ganz zufällig - analog zu dem klassischen sozialwissenschaftlichen Schisma zwischen "Quantis“ und „Qualis“.

Im Sinne der beiden Sammelbände der 1980er zu regionaler politischer Kultur streichen der qualitativ-hermeneutischen Tradition verpflichtete Autoren deutlich seltener Meinungen, Einstellungen und Werte heraus, sondern vielmehr Mentalitäten, Identitäten, Traditionsbestände, Symbole, Sprache und ,ungeschriebene Verfassungen“" (Rohe 1994: 5) in den Ländern, die sie vornehmlich - aber nicht ausschließlich - separat in den Blick nehmen. Anders als die quantitativ-umfragebasierte Forschung erhielt dieser Bereich in den letzten Jahren Auftrieb. So zollt die Politikwissenschaft in unregelmäßigen Abständen den Regionalkulturen in Baden-Württemberg (Langewiesche/Steinbach 2008; Wehling 2006; Weinacht 2012), Bayern (Glaab/Weigl 2013), Hessen (Schissler 1998), Nordrhein-Westfalen (Korte/Florack/Grunden 2006: 26 ff.) und Rheinland-Pfalz ${ }^{9}$ ihre Aufmerksamkeit. Nur ist dies schwer zu erkennen, verbergen sich doch politisch-kulturelle Porträts häufig in Landeskunden (,Das politische System in ...",,Politik in ..."), wo sie einen Aspekt der Landespolitik repräsentieren. Gleichwohl: Vom unlängst gesteigerten Interesse der Politikwissenschaft an den Ländern hat der Analysegegenstand politische Kultur gleichfalls profitiert - wenn auch in überschaubaren Ausmaßen.

Die Erosion des noch zu Beginn der 1990er Jahre vorherrschenden Ungleichgewichts bei der Betrachtung von Ländern aus dem Osten und dem Westen ist längst im Gange. So bedienen mittlerweile auch in den „,neuen“ Ländern vordringlich Landeskunden die wissenschaftliche Neugier an Mentalitäten und Iden- 
titäten - etwa zu Mecklenburg-Vorpommern (Huchel 2013; Werz 2006), Sachsen (Gerlach 1993; Jesse/Schubert/Thieme 2014: 287 ff.) und Sachsen-Anhalt (Holtmann/Boll 2012: 203 ff.). Drei Unterschiede gibt es dennoch. Erstens: Die Binnendifferenzierung einzelner Länder sowie lokale Identitäten und Mentalitäten erfahren im Westen eine größere (wenngleich immer noch geringe) Aufmerksamkeit als im Osten. ${ }^{10}$ Zweitens: Im Schatten der sozialistischen Vergangenheit fragte die Politikwissenschaft vor allem in den letzten Jahren nach der demokratischen Reife der Ostdeutschen - aus verständlichen Gründen fehlt eine solche Forschung im Westen. Dass die ostdeutschen Landesregierungen zu solchen Lagebildern häufig den Auftrag erteilten, erklärt, warum die Studien auf Bundesländer zugeschnitten sind. ${ }^{11}$ Drittens: Die politischen Kulturen ostdeutscher Länder gerieten - zumal in den 1990ern - häufiger in einen vergleichenden Blick als westdeutsche - hier schimmert das Interesse an den mentalen Hinterlassenschaften der DDR durch, die gerade während der politisch-gesellschaftlich-wirtschaftlichen Transformation noch virulent waren. ${ }^{12}$ Gleichwohl: Dieser Forschungstrend ist mittlerweile wieder rückläufig.

Aufschlussreich an dem äußerst heterogenen Forschungsstand zu politischen Landeskulturen und Mentalitäten in Deutschland ist weniger die Vielzahl an Einblicken, sondern ihre geografische Konzentration. So macht die Wissenschaft einzelnen Ländern in gewisser Regelmäßigkeit die Aufwartung (etwa Baden-Württemberg, Bayern, Rheinland-Pfalz), andere Landstriche sind hingegen nach wie vor terra incognita (so Berlin, Brandenburg, Niedersachsen, Schleswig-Holstein). An der Wurzel dieses Problems liegt die Abhängigkeit des Forschungszweiges von den wissenschaftlichen Präferenzen Einzelner: So gäbe es etwa keinen nennenswerten Kenntnisstand zu Baden-Württemberg, Hessen und Thüringen ohne Hans-Georg Wehling, Jakob Schissler und Karl Schmitt. Solange aber die qualitativ-historische Regionale-Politische-Kultur-Forschung an die Agenda von diesem und jenem gebunden ist, ruht ihre Eigenständigkeit auf tönernen Füßen. Das unterstreicht die Qualität der Publikationen, über die deren schiere Anzahl nicht hinwegtäuschen darf: Von einigen komparativen Studien abgesehen, handelt es sich fast durchweg um einführende, unsystematische und über Skizzen keinesfalls hinausweisende Überblicksaufsätze. Ganzheitliche Betrachtungen - am ehesten noch für Baden-Württemberg, Hessen und Rheinland-Pfalz zu finden - stehen für die Mehrheit der Länder aus. Und selbst hier hält „,politische Kultur“ leider allzu häu-

10 Zu Baden-Württemberg Kießling/Schiersner 2009; zu Bayern Weigl/Zöhrer 2005.

11 Zu Brandenburg Jaschke 2006; zu Thüringen Edinger/Hallermann/Schmitt 2004; Schmitt 2011; Best u. a. 2013; zu Sachsen Berth u. a. 2012; Schöppner/Sagurna 1995.

12 Blancke/Tiemann 1993; Kreikenbom/Stapelfeld 1995; Yoder 1998; Yoder 2000. 
fig als „Klammerbegriff“ für Parteiensystemtrends, direktdemokratische Verfahren, Verwaltungsstrukturen und Massenmedien her - ein catch all term par excellence.

\section{Regionale politische Kultur seit der Wiedervereinigung: ein brachliegen- des Forschungsfeld (?)}

Ein Vierteljahrhundert nach der Wiedervereinigung leidet „regionale politische Kultur" - vor allem im geisteswissenschaftlich-verstehenden Forschungsbereich unter einem Imageproblem: Das Thema darf zwar - der Vollständigkeit halber bei Landeskunden und Einführungen nicht fehlen, ihm eigenständige Analysen zu widmen trägt aber kaum wissenschaftliche Lorbeeren ein. Darum stößt, wer sich auf aktuellem Forschungsstand zu jenen tradierten Riten, Mentalitäten und Symbolen vertieft informieren will, in die landespolitische Praxis eingebettet ist und ohne die diese vielfach schlechterdings ein Buch mit sieben Siegeln bleibt, häufig auf Lücken - etwa, wenn es um die Dominanz der CSU in Bayern, ${ }^{13}$ variierendes Wahlverhalten im „Ländle“ (Glück 2003) oder den Unterschied zwischen dem „,roten“ Brandenburg und dem ,schwarzen“ Sachsen geht.

Angesichts solcher Fragen frappiert das Nischendasein des Forschungszweigs. Besonders gering (bzw. veraltet) sind wissenschaftlich fundierte Kenntnisse zu den Mentalitäten und Identitäten an der Küste (Bremen, Hamburg, Niedersachsen, Schleswig-Holstein) und im Osten der Republik (Berlin, Brandenburg, Sachsen, Sachsen-Anhalt). An eine vergleichende Gesamtdarstellung aller politischen Regionalkulturen ist daher gegenwärtig nicht zu denken, von einer den Wandel aufspürenden Längsschnittanalyse ganz zu schweigen.

Ein Erschwernis, das einer Bedeutungssteigerung des Themenfeldes im Wege steht, ist der inflationäre Gebrauch des Terminus: Dient (regionale) politische Kultur als terminologische „Resterampe“ der Politikwissenschaft, braucht das Fehlen vergleichender Arbeiten, die auf einem präzisen, operationalisierbaren Konzept aufbauen, ebenso wenig zu verwundern wie der geringe Status innerhalb der Disziplin.

Was subnational vergleichende, quantitative Ansätze angeht, so beruht ihre größte Hürde in der Abhängigkeit von der Umfrageforschung, deren beliebteste Analyseebenen immer noch der Nationalstaat und Ost/West sind. Schlüsse auf Länderebene sind aufgrund der geringen Fallzahlen daher nur zum Preis großer Vertrauensbereiche zu haben. Variierende Themenschwerpunkte und Items ver- 
sperren den Ausweg, den die Verknüpfung von Einzelfallstudien bringen könnten. Dennoch zeichnet sich ein Umdenken ab. Davon zeugen etwa zwei Studien der Bertelsmann-Stiftung zu föderalismus- und gesellschaftsbezogenen Einstellungen in den Ländern - eine davon primäranalytisch (2008), die andere sekundäranalytisch (2014).

Voraussetzung für subnationale Politische-Kultur-Vergleiche, die Schlüsse auf die Landesbevölkerungen zu vertretbaren Vertrauensbereichen zulassen sollen, sind quantitative Surveys mit einer Mindestfallzahl von 200 bis 300 pro Bundesland. Sicher: Derartige Umfragen mit 3.000 bis 5.000 realisierten Interviews haben ihren Preis. Allerdings können Analysen auf Bundes- bzw. Ost-West-Ebene auf das Datenmaterial gleichfalls zurückgreifen.

Ein weniger erhebungstechnisches als inhaltliches Desiderat stellt der Zusammenhang von Meinungen, Einstellungen und Werten zu verschiedenen Ebenen des politischen Systems dar. So ist etwa nicht geklärt, wie Meinungen zum politischen System eines Bundeslandes jene zum politischen System auf Bundesebene beeinflussen - und umgekehrt. Hier offenbart sich ein geradezu jungfräuliches Forschungsgebiet, das die Regionale-Politische-Kultur-Forschung - zumindest in Deutschland - bislang gemieden hat.

Schließlich: Die Regionale-Politische-Kultur-Forschung ist ein Spiegel des gesamten Faches, in dem qualitative und quantitative Zugänge nur bedingt einander rezipieren. Auf Umfragen basierende Studien greifen auf Traditionsbestände, Symbole und Riten meist nur dann zurück, wenn es gilt, spezifische Befunde in ein großes Ganzes einzubetten. Umgekehrt dient geisteswissenschaftlich-verstehenden Ansätzen die Demoskopie häufig nur als Hilfswissenschaft, mit der sich die eigenen Eindrücke allenfalls unterstreichen lassen, die aber ansonsten als oberflächlich abgetan wird. Dabei entfalten Fragen der folgenden Art, die nur durch eine Verknüpfung beider Forschungsstränge zu beantworten sind, enormes Potenzial: Wie kommen - trotz augenscheinlich unterschiedlicher politischer Mentalitäten und Traditionen - ähnliche normative Vorstellungen von einem ,guten" demokratischen Verfassungsstaat etwa in Baden-Württemberg, Bayern, Rheinland-Pfalz und Hessen sowie in Niedersachsen, Nordrhein-Westfalen, Schleswig-Holstein und Thüringen zustande? Welche Wertebestände fördern eine gemeinsame regionale Identität (wie etwa am 9. Dezember 1951 in Baden, Württemberg-Baden und Württemberg-Hohenzollern), welche verhindern eine Identifikation (wie in Berlin und Brandenburg am 5. Mai 1996)? Wie wirkt sich eine gemeinsame Identität auf die Herausbildung gemeinsamer Normen aus?

Die beispielhaften Fragen zeigen, wie wenig ausdifferenziert die Forschung zu regionaler politischer Kultur noch ist. Die Politikwissenschaft ist gut beraten, sich 
ihr zu- und von dem wenig fruchtbaren Ost-West-Vergleich abzuwenden - nicht, weil es keine „zwei politischen Kulturen“ gibt oder irgendwann einmal geben könnte, sondern weil das Forschungsdesign unangemessen ist, um der Frage nach der ,inneren Einheit“ nachzugehen, und weil es Regionalisierungstrends nicht aufspüren kann. Darum muss es die mit politisch-kultureller Vielfalt einhergehenden Herausforderungen für den Staat übersehen. Nicht zuletzt deshalb ist es für die Politikwissenschaft von Interesse, eine vergleichende Regionale-PolitischeKultur-Forschung in Deutschland zu etablieren, die ihren Namen verdient. Erste Ansätze dazu sind ein Vierteljahrhundert nach der Wiedervereinigung zu erkennen.

\section{Literatur}

Ahbe, Thomas/Gibas, Monika, 2000: Der Osten im wiedervereinigten Deutschland, in: Wolfgang Thierse/Ilse Spittmann-Rühle/Johannes L. Kuppe (Hrsg.), Zehn Jahre Deutsche Einheit, Opladen, 23-38.

Alm, Leslie R./Burkhart, Ross E./Patton, W. David/Weatherby, James B., 2001: Intrastate Regional Differences in Political Culture: A Case Study of Idaho, in: State and Local Government Review 33 (2), 109-119.

Almond, Gabriel/Verba, Sidney, 1965: The Civic Culture. Political Attitudes and Democracy in Five Nations, Boston.

Bahrmann, Hannes/Links, Christoph, 2005: Am Ziel vorbei. Die deutsche Einheit - eine Zwischenbilanz, Berlin.

Beetz, Michael/Corsten, Michael/Rosa, Hartmut/Winkler, Torsten, 2014: Was bewegt Deutschland? Sozialmoralische Landkarten engagierter und distanzierter Bürger in Ost- und Westdeutschland, Weinheim.

Berg-Schlosser, Dirk/Schissler, Jacob (Hrsg.), 1987: Politische Kultur in Deutschland. Bilanz und Perspektiven der Forschung (PVS Sonderheft 18), Opladen.

Bertelsmann Stiftung, 2008: Bürger und Föderalismus. Eine Umfrage zur Rolle der Bundesländer, Gütersloh.

Bertelsmann Stiftung, 2014: Radar gesellschaftlicher Zusammenhalt. Messen was verbindet, Gütersloh.

Berth, Hendrik/Brähler, Elmar/Zenger, Markus/Stöbel-Richter, Yve (Hrsg.), 2012: Innenansichten der Transformation. 25 Jahre Sächsische Längsschnittstudie (1987-2012), Gießen.

Best, Heinrich/Dwars, Daniel/Salheiser, Axel/Salomo, Katja, 2013: „Wie leben wir? Wie wollen wir leben?" - Zufriedenheit, Werte und gesellschaftliche 
Regionale politische Kultur zwischen Symbolanalysen und Surveydaten

Orientierungen der Thüringer Bevölkerung. Ergebnisse des Thüringen-Monitors 2013, Jena.

Beyme, Klaus von/Niedermayer, Oskar (Hrsg.), 1994: Politische Kultur in Ostund Westdeutschland, Berlin.

Blancke, Susanne/Tiemann, Heinrich, 1993: Regionale Kulturen in den neuen Bundesländern, in: Die neue Gesellschaft/Frankfurter Hefte 40 (6), 537-543.

Blatter, Joachim, 2004: From 'Spaces of Places' to 'Spaces of Flows'? Territorial and Functional Governance in Cross-Border Regions in Europe and North America, in: International Journal of Urban and Regional Research 28 (3), 530-548.

Breton, Raymond, 1970: Institutional Completeness of Ethnic Communities and the Personal Relations of Immigrants, in: American Journal of Sociology 70 (2), 193-205.

Bundesministerium für Wirtschaft und Energie (Hrsg.), 2015: Sind wir ein Volk? 25 Jahre friedliche Revolution und Deutsche Einheit, Berlin.

Caramani, Daniele/Wagemann, Claudius, 2005: A Transnational Political Culture? The Alpine Region and its Relationship to European Integration, in: German Politics 14 (1), 74-94.

Chernilo, Daniel, 2006: Social Theory's Methodological Nationalism. Myth and Reality, in: European Journal of Social Theory 9 (1), 5-22.

Cohen, Anthony P., 1996: Personal nationalism: a Scottish view of some rites, rights, and wrongs, in: American Ethnologist 23 (4), 802-815.

Easton, David, 1965 a: A Framework for Political Analysis, Englewood Cliffs.

Easton, David, 1965 b: A Systems Analysis of Political Life, New York.

Easton, David, 1975: A Re-Assessment of the Concept of Political Support, in: British Journal of Political Science 5 (4), 435-457.

Edinger, Michael/Hallermann, Andreas/Schmitt, Karl, 2004: Politische Kultur in Ostdeutschland. Die Unterstützung des politischen Systems am Beispiel Thüringens, Frankfurt a. M./New York.

Elkins, David J./Simeon, Richard E. B., 1974: Regional Political Cultures in Canada, in: Canadian Journal of Political Science 7 (4), 397-437.

Falter, Jürgen $W$., 1982: Bayerns Uhren gehen wirklich anders. Politische Verhaltens- und Einstellungsunterschiede zwischen Bayern und dem Rest der Bundesrepublik, in: ZParl 13 (4), 504-520.

Falter, Jürgen W., 1988: Wie gehen sie denn nun wirklich, die bayerischen Uhren?, in: ZParl 19 (1), 113-114. 
Falter, Jürgen W./Gabriel, Oscar W./Rattinger, Hans (Hrsg.), 2000: Wirklich ein Volk? Die politischen Orientierungen von Ost- und Westdeutschen im Vergleich, Opladen.

Feist, Ursula/Liepelt, Klaus, 1994: Auseinander oder miteinander? Zum unterschiedlichen Politikverständnis der Deutschen in Ost und West, in: Max Kaase/Hans-Dieter Klingemann (Hrsg.), Wahlen und Wähler, Opladen, 575-611.

Freitag, Markus/Fritz, David/Vatter, Adrian, 2010: Vergleichende subnationale Analysen für Deutschland. Eine Einführung, in: Markus Freitag/Adrian Vatter (Hrsg.), Vergleichende subnationale Analysen für Deutschland. Institutionen, Staatstätigkeiten und politische Kulturen, Münster, 7-26.

Freitag, Markus/Heinsohn, Till, 2011: Auch Heterogenität zwischen den Ländern lässt sich zutage fördern. Die neuen Bundesländer aus vergleichender subnationaler Perspektive, in: Astrid Lorenz (Hrsg.), Ostdeutschland und die Sozialwissenschaften, Opladen, 401-418.

Freitag, Markus/Traunmüller, Richard, 2008: Sozialkapitalwelten in Deutschland. Soziale Netzwerke, Vertrauen und Reziprozitätsnormen im subnationalen Vergleich, in: ZfVP 2 (1), 221-256.

Freitag, Markus/Vatter, Adrian (Hrsg.), 2010: Vergleichende subnationale Analysen für Deutschland. Institutionen, Staatstätigkeiten und politische Kulturen, Münster.

Fuchs, Dieter, 1989: Die Unterstützung des politischen Systems der Bundesrepublik Deutschland, Opladen.

Fuchs, Dieter/Gabriel, Oscar W./Völkl, Kerstin, 2002: Vertrauen in politische Institutionen und politische Unterstützung, in: ÖZP 31 (4), 427-450.

Fuchs, Dieter/Roller, Edeltraud/Weßels, Bernhard (Hrsg.), 2002: Bürger und Demokratie in Ost und West. Studien zur politischen Kultur und zum politischen Prozess, Wiesbaden.

Fuchs, Dieter/Roller, Edeltraud/Weßels, Bernhard, 1997: Die Akzeptanz der Demokratie des vereinigten Deutschland. Oder: Wann ist ein Unterschied ein Unterschied?, in: APuZ, B 51, 3-12.

Gabriel, Oscar W., 1987: Politische Kultur, Postmaterialismus und Materialismus in der Bundesrepublik Deutschland, Opladen.

Gabriel, Oscar W., 1994, Lokale politische Kultur, in: Ulrich von Alemann/Kay Loss/Gerhard Vowe (Hrsg.), Politik, Wiesbaden, 201-252.

Gabriel, Oscar W., 2005: Politische Einstellungen und politische Kultur, in: ders./ Everhard Holtmann (Hrsg.), Handbuch Politisches System der Bundesrepublik Deutschland, München (3. Aufl.), 457-523. 
Regionale politische Kultur zwischen Symbolanalysen und Surveydaten

Gabriel, Oscar W./Falter, Jürgen W./Rattinger, Hans (Hrsg.), 2005: Wächst zusammen, was zusammengehört? Stabilität und Wandel politischer Einstellungen im wiedervereinigten Deutschland, Baden-Baden.

Gerlach, Siegfried, 1993: Sachsen. Eine politische Landeskunde, Stuttgart.

Glaab, Manuela/Weigl, Michael, 2013: Politik und Regieren in Bayern. Rahmenbedingungen, Strukturmerkmale, Entwicklungen, in: dies. (Hrsg.), Politik und Regieren in Bayern, Wiesbaden 2013, 19-98.

Glück, Horst, 2003: Neue Tendenzen und alte Traditionen: Das Wahlverhalten in Baden-Württemberg bei der Landtagswahl 2001, in: Andreas Dornheim/ Sylvia Greiffenhagen (Hrsg.), Identität und politische Kultur, Stuttgart, 203-212.

Greiffenhagen, Martin/Greiffenhagen, Sylvia, 1993: Ein schwieriges Vaterland. Zur politischen Kultur im vereinigten Deutschland, München.

Greiffenhagen, Martin/Greiffenhagen, Sylvia, 2000: Zwei politische Kulturen? Wissenschaftliche und politische Unsicherheiten im Umgang mit der deutschen Vereinigung, in: Hans-Georg Wehling (Hrsg.), Deutschland Ost Deutschland West, Stuttgart, 179-185.

Henderson, Ailsa, 2007: The Regionalisation of Political Culture. Paper presented at the 2007 CPSA Annual Conference, CPSA Homepage, Ottawa, http:// www.cpsa-acsp.ca/papers-2007/Henderson.pdf (Stand: 29.1.15).

Henderson, Ailsa, 2010 a: „Small Worlds“ as Predictors of General Political Attitudes, in: Regional \& Federal Studies 20 (4-5), 469-485.

Henderson, Ailsa, 2010 b: Why Regions Matter: Substate Polities in Comparative Perspective, in: Regional and Federal Studies 20 (4-5), 439-445.

Hollenstein, Oliver, 2012: Das doppelt geteilte Land. Neue Einblicke in die Debatte über West- und Ostdeutschland, Wiesbaden.

Holtmann, Everhard/Boll, Bernhard, 1997: Sachsen-Anhalt. Eine politische Landeskunde, Opladen (2. Aufl.).

Huchel, Philipp, 2013: Politische Kultur in Mecklenburg-Vorpommern, in: Martin Koschkar/Christian Nestler/Christopher Scheele (Hrsg.), Politik in Mecklenburg-Vorpommern, Wiesbaden, 203-228.

Inglehart, Ronald, 1977: The Silent Revolution. Changing Values and Political Styles Among Western Publics, Princeton.

Inglehart, Ronald, 1989: Kultureller Umbruch. Wertewandel in der westlichen Welt, Frankfurt a. M./New York.

Inglehart, Ronald/Carballo, Marita, 1997: Does Latin America Exist? (And is There a Confucian Culture?): A Global Analysis of Cross-Cultural Differences, in: PS: Political Science and Politics 30, 34-47. 
Jaschke, Hans-Gerd, 2006: Analyse der politischen Kultur Brandenburgs im Hinblick auf ihre demokratiestützende oder demokratieproblematische Wirkung. Im Auftrag der Enquete-Kommission „Aufarbeitung der Geschichte und Bewältigung von Folgen der SED-Diktatur und des Übergangs in einen demokratischen Rechtsstaat im Land Brandenburg" des Landtags Brandenburg, Potsdam.

Jeffery, Charlie/Wincott, Daniel, 2010: The Challenge of Territorial Politics: Beyond Methodological Nationalism, in: Colin Hay (Hrsg.), New Directions in Political Science, Houndsmill, 167-188.

Jesse, Eckhard, 2008: Zwei verschiedene politische Kulturen in Deutschland?, in: Uwe Backes/Alexander Gallus (Hrsg.), Demokratie in Deutschland, Köln/ Weimar/Wien, 166-192.

Jesse, Eckhard/Schubert, Thomas/Thieme, Tom, 2014: Politik in Sachsen, Wiesbaden.

Keil, Silke I./Deth, Jan van (Hrsg.), 2012: Deutschlands Metamorphosen. Ergebnisse des European Social Survey 2002 bis 2008, Baden-Baden.

Kießling, Rolf/Schiersner Dietmar (Hrsg.), 2009: Erinnerungsorte in Oberschwaben. Regionale Identität im kulturellen Gedächtnis, Konstanz.

Klages, Helmut/Gensicke, Thomas, 1992: Wertewandel in den neuen Bundesländern. Fakten und Deutungsmodelle, in: Wolfgang Glatzer/Heinz-Herbert Noll (Hrsg.), Lebensverhältnisse in Deutschland, Frankfurt a. M., 301-326.

Korte, Karl-Rudolf/Florack, Martin/Grunden, Timo, 2006: Regieren in Nordrhein-Westfalen. Strukturen, Stile und Entscheidungen 1990-2006, Wiesbaden.

Kreikenbom, Henry/Stapelfeld, Maxi, 1995: Die Transformation der politischen Kultur in den neuen Bundesländern am Beispiel der Bürgerorientierung gegenüber den soziopolitischen Vermittlungsstrukturen und dem politischen System der Bundesrepublik, Halle.

Landeszentrale für politische Bildung Baden-Württemberg (Hrsg.), 1985: Regionale politische Kultur (Redaktion: Hans-Georg-Wehling), Stuttgart.

Langewiesche, Dieter/Steinbach, Peter (Hrsg.), 2008: Der deutsche Südwesten. Regionale Traditionen und historische Identitäten (FS Wehling), Stuttgart.

Lepsius, Mario R., 2009: Interessen, Ideen und Institutionen, Wiesbaden.

Lieske, Joel, 2010: The Changing Regional Subcultures of the American States and the Utility of a New Cultural Measure, in: Political Research Quarterly 63 (3), 538-552.

Lipset, Seymour Martin, 1963: Political Man. The Social Bases of Politics, London. 
Regionale politische Kultur zwischen Symbolanalysen und Surveydaten

Mannewitz, Tom, 2012: It's the region, stupid. Deutschlands politische Kultur nach der staatlichen Einheit, in: MUT 47 (10), 60-69.

Mannewitz, Tom, 2013: Kein Ost-West-Gegensatz: Regionale Unterschiede der deutschen politischen Kultur, in: Gesellschaft - Wirtschaft - Politik 62 (2), 205-215.

Mannewitz, Tom, 2015: Politische Kultur und demokratischer Verfassungsstaat. Ein subnationaler Vergleich zwei Jahrzehnte nach der deutschen Wiedervereinigung, Baden-Baden, i. E.

Martins, Herminio, 1974: Time and Theory in Sociology, in: John Rex (Hrsg.), Approaches to Sociology, London, 246-294.

McGrane, David/Berdahl, Loleen, 2013: ,Small Worlds“ No More: Reconsidering Provincial Political Cultures in Canada, in: Regional and Federal Studies 23 (4), 479-493.

Meulemann, Heiner (Hrsg.), 1998: Werte und nationale Identität im vereinten Deutschland. Erklärungsansätze der Umfrageforschung, Opladen.

Mintzel, Alf, 1985: Das traditionskräftige und staatlich selbstbewußte Bayern. Bayerns politische Kultur im Wandel, in: Landeszentrale für politische Bildung Baden-Württemberg 1985, 146-162.

Mintzel, Alf, 1987 a: Besonderheiten der politischen Kultur Bayerns. Facetten und Etappen einer politisch-kulturellen Homogenisierung, in: Dirk Berg-Schlosser/ Jacob Schissler (Hrsg.), Politische Kultur in Deutschland. Bilanz und Perspektiven der Forschung (PVS Sonderheft 18), Opladen, 295-308.

Mintzel, Alf, 1987 b: Gehen Bayerns Uhren wirklich anders?, in: ZParl 18 (1), 77-93.

Patzelt, Werner J., 2008: Demokratievertrauen und Demokratieakzeptanz in den neuen Ländern, in: Bernhard Vogel (Hrsg.), Politische Kultur in den neuen Ländern, Cadenabbia, 7-36.

Pickel, Gert, 2002: Jugend und Politikverdrossenheit. Zwei politische Kulturen im Deutschland nach der Vereinigung?, Opladen.

Pickel, Susanne, 2011: Erst Vielfalt schafft Wissen über Ost und West. Strukturdaten, Surveys, qualitative Interviews und Methodenmix, in: Astrid Lorenz (Hrsg.), Ostdeutschland und die Sozialwissenschaften, Opladen, 375-400.

Pickel, Susanne/Pickel, Gert, 2006: Politische Kultur- und Demokratieforschung. Grundbegriffe, Theorien, Methoden, Wiesbaden.

Pickel, Susanne/Pickel, Gert/Walz, Dieter (Hrsg.), 1998: Politische Einheit, kultureller Zwiespalt? Die Erklärung politischer und demokratischer Einstellungen in Ostdeutschland vor der Bundestagswahl 1998, Frankfurt a. M. 
Pollack, Detlef, 1999: Support for Democracy in Eastern and Western Germany: an Attempt to Explain the Differences, in: European Journal of Sociology 45 (2), 257-272.

Putnam, Robert D., 1993: Making Democracy Work. Civic Traditions in Modern Italy, Princeton.

Reisinger, William M., 1995: The Renaissance of a Rubric. Political Culture as Concept and Theory, in: International Journal of Public Opinion Research 7 (4), 328-352.

Reissig, Rolf, 2000: Die gespaltene Vereinigungsgesellschaft. Bilanz und Perspektiven der Transformation Ostdeutschlands und der deutschen Vereinigung, Berlin.

Rohe, Karl, 1987: Politische Kultur und der kulturelle Aspekt von politischer Wirklichkeit - Konzeptionelle und typologische Überlegungen zu Gegenstand und Fragestellung Politischer Kultur-Forschung, in: Dirk Berg-Schlosser/ Jacob Schissler (Hrsg.), Politische Kultur in Deutschland. Bilanz und Perspektiven der Forschung (PVS Sonderheft 18), Opladen, 39-48.

Rohe, Karl, 1990: Politische Kultur und ihre Analyse. Probleme und Perspektiven der politischen Kulturforschung, in: Historische Zeitschrift 250 (2), 321-346.

Rohe, Karl, 1994: Politische Kultur: Zum Verständnis eines theoretischen Konzepts, in: Klaus von Beyme/Oskar Niedermayer (Hrsg.), Politische Kultur in Ost- und Westdeutschland, Berlin, 1-21.

Rohrschneider, Robert, 1999: Learning Democracy: Democratic and Economic Values in Unified Germany, Oxford.

Salzborn, Samuel/Diehl, Paula (Hrsg.), 2013: Politische Theorie(n) der Politischen Kultur, ZPTh 4 (2), Opladen.

Sarcinelli, Ulrich/Falter, Jürgen W./Mielke, Gerd (Hrsg.), 2000: Politische Kultur in Rheinland-Pfalz, Mainz.

Sarcinelli, Ulrich/Falter, Jürgen W./Mielke, Gerd/Benzner, Bodo (Hrsg.), 2010: Politik in Rheinland-Pfalz. Gesellschaft, Staat und Demokratie, Wiesbaden.

Schissler, Jakob (Hrsg.), 1998: Politische Kultur und politisches System in Hessen, Frankfurt a. M.

Schmitt, Karl, 2011: Politische Kultur in Thüringen: zehn Jahre Thüringen-Monitor, in: Andreas Eis/Torsten Oppelland/Christian K. Tischner (Hrsg.), Politik kulturell verstehen, Schwalbach/Ts., 29-43.

Schneider, Christoph, 2013: Regionale Unterschiede der politischen Kultur in Deutschland und Europa, Frankfurt a. M.

Schöppner, Klaus-Peter/Sagurna, Michel, 1995: Sächsische Meinungsbilder. Die ersten Jahre Freistaat Sachsen im Spiegel der Demoskopie, Dresden. 
Regionale politische Kultur zwischen Symbolanalysen und Surveydaten

Schroeder, Klaus, 2000: Der Preis der Einheit. Eine Bilanz, München.

Sturm, Roland, 2004: Politische Kultur, in: Ludger Helms/Uwe Jun (Hrsg.), Politische Theorie und Regierungslehre, Frankfurt a. M., 302-323.

Veen, Hans-Joachim, 2012: Wie viel Einheit brauchen wir? Die ,innere Einheit“ zwischen Gemeinschaftsmythos und neuer Vielfalt, in: Eckhard Jesse (Hrsg.), Eine normale Republik?, Baden-Baden, 119-136.

Veen, Hans-Joachim, 2013: Eine Jugend in Deutschland? Orientierungen und Verhaltensweisen der Jugend in Ost Und West, Wiesbaden.

Wehling, Hans-Georg, 2006: Baden-Württemberg. Zur Geschichte eines jungen Bundeslandes, in: ders./Reinhold Weber (Hrsg.), Baden-Württemberg. Gesellschaft, Geschichte, Politik, Stuttgart, 9-32.

Weigl, Michael/Zöhrer, Michaela, 2005: Regionale Selbstverständnisse und gegenseitige Wahrnehmung von Deutschen und Tschechen, München.

Weinacht, Paul-Ludwig, 2012: Politische Kultur am Oberrhein. Studien zur Geschichte Badens, Karlsruhe.

Werz, Nikolaus, 2006: Politik und Bürger. Demokratie und Verfassung seit 1989, in: Landeszentrale für politische Bildung Mecklenburg-Vorpommern (Hrsg.), Politische Landeskunde Mecklenburg-Vorpommern, Schwerin, 51-66.

Westle, Bettina, 2009: Politische Kulturen, Globalisierung und Europäische Union, in: Oscar W. Gabriel/dies. (Hrsg.), Politische Kultur, Baden-Baden, 529-578.

Yoder, Jennifer A., 1998: The Regionalization of Political Culture and Identity in Post-Communist Eastern Germany, in: East European Quarterly 32 (2), 197-219.

Yoder, Jennifer A., 2000: West-East Integration: Lessons from East Germany's Accelerated Transition, in: East European Politics \& Societies 14 (2), 114-138.

Korrespondenzanschrift:

Jun.-Prof. Dr. phil. habil. Tom Mannewitz

Institut für Politikwissenschaft

Juniorprofessur Politikwissenschaftliche Forschungsmethoden

Technische Universität Chemnitz

Thüringer Weg 9

09126 Chemnitz

E-Mail: Tom.Mannewitz@phil.tu-chemnitz.de 